\title{
Unsteady flow inferred for Thwaites Glacier, and comparison with Pine Island Glacier, West Antarctica
}

\author{
Jonathan BAMBER, ${ }^{1}$ Eric RIGNOT ${ }^{2}$ \\ ${ }^{1}$ Centre for Polar Observations and Modelling, Bristol Glaciology Centre, University of Bristol, University Road, Bristol BSo 1SS, England \\ E-mail:j.l.bamber@bristol.ac.uk \\ 2 Jet Propulsion Laboratory, California Institute of Technology, 4800 Oak Grove Drive, Pasadena, California 91109-8099, U.S.A.
}

\begin{abstract}
We present a comparison of surface velocities in 1996, derived from interferometric synthetic aperture radar, with an estimate of the long-term, depth-averaged velocity based on the assumption of steady-state flow for both Pine Island Glacier and its neighbour, Thwaites Glacier, West Antarctica. The results show that the former was close to balance conditions in 1996 (within 9\%). The ice flux and velocity of the latter is significantly different in magnitude and distribution from that required to maintain the basin in a state of mass balance over the long term. The balance flux was found to be $32 \pm 19 \%$ less than the measured outgoing flux. We conclude that the mass imbalance and dramatic difference in the pattern of flow is most likely due to a substantial change in the flow regime of Thwaites Glacier in the recent past.
\end{abstract}

\section{INTRODUCTION}

Pine Island and Thwaites Glaciers drain the sector of the West Antarctic ice sheet (Fig. 1) believed by some to be most at risk of rapid change and potential collapse (Hughes, 1981; Vaughan and others, 2001). Early studies of the mass balance of Pine Island Glacier (PIG) suggested massive thickening in this region (Bentley and Giovinetto, 1991), yet with considerable uncertainty in the data. This result was challenged by Lucchitta and others (1994) with the aid of more accurate glacier velocities than used earlier. They also suggested that the location of the glacier grounding line obtained by Crabtree and Doake (1982) was in error. The grounding-line location was unequivocally detected using European Remote-sensing Satellite (ERS) interferometry (Rignot, 1998a), and was found to be about $30 \mathrm{~km}$ downstream of the Crabtree and Doake (1982) location. More importantly, ERS interferometry data revealed that the grounding line retreated rapidly $(\sim 5 \mathrm{~km})$ between 1992 and 1996, which suggested that, during that time, PIG was thinning by $3.5 \mathrm{~m} \mathrm{a}^{-1}$ at the glacier centre.

More recently, radar altimeter data collected between 1992 and 1999 showed that ice thinning prevails inland of the grounding line of PIG, along the areas of fast flow, with an average thinning rate of $1.6 \mathrm{~m} \mathrm{a}^{-1}$ (Shepherd and others, 2001). Independently, Rignot and others (2002) found that the glacier accelerated 18\% between 1992 and 2000, which explains the thinning trend observed by radar altimetry and also the grounding-line retreat. They concluded their study by suggesting that these ice-flow changes were caused by enhanced lubrication of the glacier bed. All these results taken together suggest that PIG is undergoing rapid changes and is thinning at present.

Fewer results have been obtained on neighbouring Thwaites Glacier (TWG). A recent study (Rignot, 2001) suggests, however, that its grounding line has been retreating since 1992 almost as rapidly as on PIG and that its ice discharge is $30 \pm 15 \%$ larger than required to maintain the basin in a state of mass balance. Meanwhile, the glacier velocity has not changed in the recent past for the grounded part of the glacier (Rosanova and others, 1999), but the fastflow area has widened by several kilometres between 1996 and 2000 (Rignot and others, 2002). Hence, while the pattern of retreat and thinning differs between the two glaciers, there are indications that this sector of West Antarctica is undergoing major, rapid changes.

In this study, we present a comparison of ice velocities measured using ERS interferometric synthetic aperture radar (InSAR) data with balance velocities that represent a longer-term, depth-averaged estimate of velocity based on the assumption of steady-state flow. This comparison is complementary to the studies discussed earlier, providing information on recent changes in ice flow that may have taken place in relation to the longer-term behaviour of the ice sheet.

\section{DATASETS}

\section{Balance velocities $\left(U_{\mathrm{b}}\right)$}

Here we have used a series of recently published datasets, derived from satellite remote sensing and in situ measurements, to calculate a quantity known as the balance velocity $\left(U_{\mathrm{b}}\right)$. This quantity is derived by calculating a balance flux and dividing this by the ice thickness. It was calculated using the approach proposed by Budd and Warner (1996), which is outlined below.

If $A$ is the net surface mass balance in ice equivalent units per unit area per unit time then at any point with ice thickness $Z$ and depth-averaged horizontal velocity $U_{\mathrm{b}}$, steady state is obtained if:

$$
A=\nabla\left(U_{\mathrm{b}} Z\right)
$$




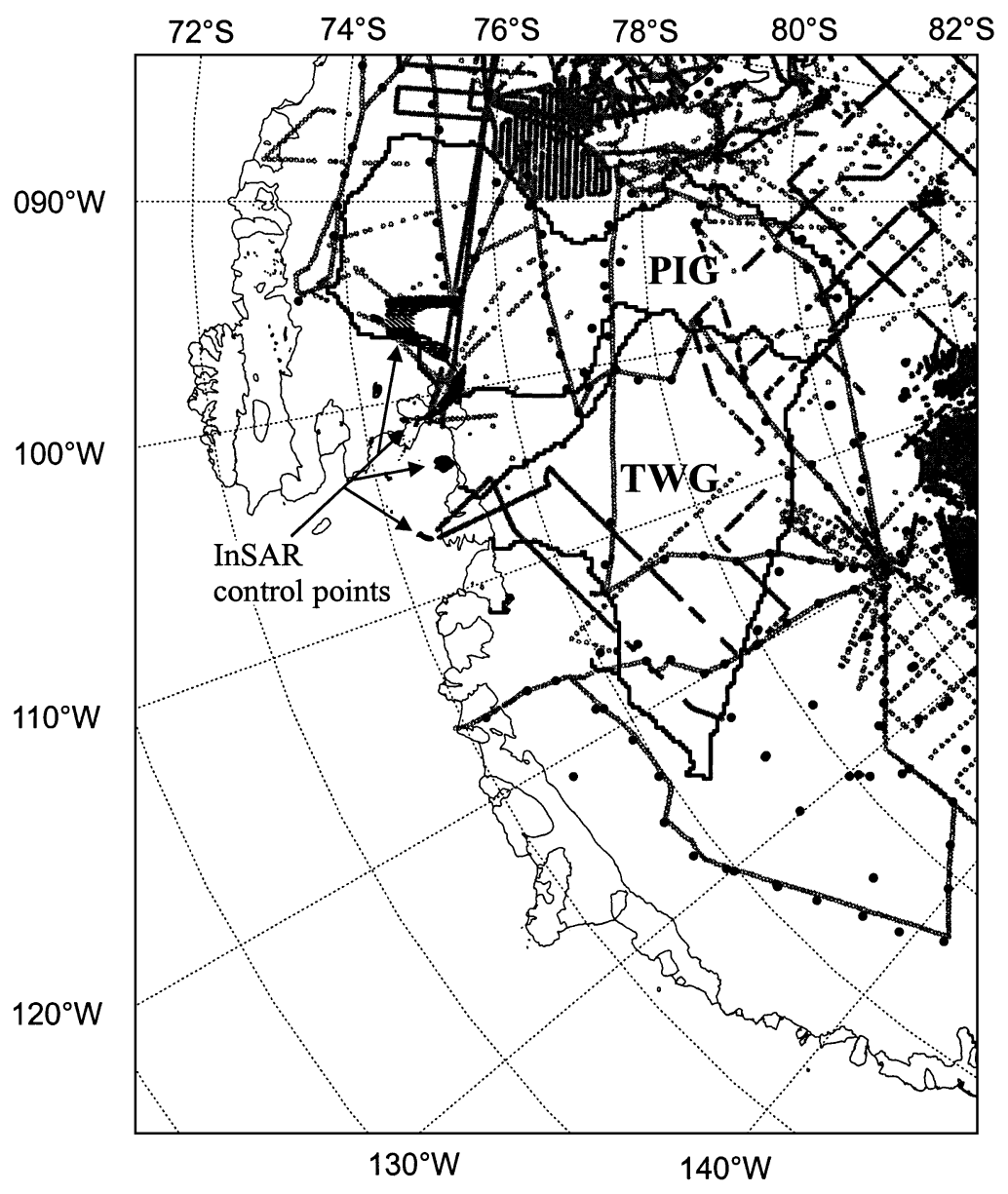

Fig. 1. Map of the area, showing the locations of in situ accumulation measurements, the glacier locations, and their catchment areas derived from a digital elevation model (DEM) of the ice sheet (Vaughan and others, 2001). The coverage of airborne radio-echo sounding (RES) measurements is shown by grey lines, and spot estimates of ice thickness by circles. The larger solid circles indicate the location of accumulation measurements. Also shown are the areas used as velocity control for the interferometry.

$U_{\mathrm{b}}$ is consequently the horizontal vector flux density in the direction of flow divided by the ice thickness:

$$
U_{\mathrm{b}}=\frac{\Phi}{Z},
$$

where $\Phi(x, y)$ is the balance flux distribution. For any closed area, $S$ (in this case the drainage basins of PIG and TWG), the total gain of mass through the upper or lower surface is balanced by the net outward flow through the boundary:

$$
\iint_{s}(A-M) \partial x \partial y=\int_{c} \bar{U}_{\mathrm{b}} Z n \partial l=\Psi,
$$

where $\partial l$ is an elemental length along the boundary $C$ of the surface, $n$ is the normal to the boundary and $\Psi$ is the scalar flux integral (or volume flux) across the boundary $C$. Here a finite-difference scheme was used on a quadrilateral grid allowing the balance flux distribution to be separated into its $x$ and $y$ components based on the slope direction, $\theta$ :

$$
\begin{aligned}
& \phi_{x}=\phi \cos \theta \\
& \phi_{y}=\phi \sin \theta,
\end{aligned}
$$

where

$$
\phi=|\Phi| .
$$

The computer scheme used to calculate the scalar fluxes has been presented in detail elsewhere (Budd and Warner, 1996), and only the most relevant points are considered here. The key to the scheme is that rather than calculating vector flux distribution, $\Phi$, the total scalar flux, $\Psi$, is calculated for each gridpoint by discretizing Equation (3) in the form:

$$
\Psi_{i, j}^{(\text {out })}=A_{i, j} \Delta x \Delta y+\Psi_{i, j}^{(\text {in })},
$$

where $\Psi^{(\text {out })}$ and $\Psi^{(\text {in })}$ are the outgoing and incoming total scalar fluxes respectively.

A centred difference approach was used to estimate slope and flux vectors, which means that for a point $x_{i, j}$ only the four surrounding points, $x_{i-1, j} x_{i, j-1} x_{i+1, j} x_{i, j+1}$ are relevant to the properties of $x_{i, j}$. Thus flux from $x_{i, j}$ can only flow into or out of one of these four points, and $\Psi^{(i n)}$ is determined by the slope vector calculated using the four surrounding points and the magnitude of $\Psi^{\text {(out) }}$ in the upstream gridpoints. Thus, in principle, flux should be conserved. This is the case except in the vicinity of bedrock outcrops, where the scheme does not always avoid flow into an area of zero ice thickness. This is, however, a minor effect, and comparison of the net mass balance, $A$, with the flux crossing the boundary of $S$ indicates that flux is conserved to better than $0.1 \%$. The key to the scheme is the sorting of gridpoints in order of descending elevation, so that the total flux into a point is known from the contribution of upslope points that have already been processed. This allows for a simple calculation of the flux integral leaving any gridpoint $x_{i, j}$. For points with equal elevation, their sorted order depends on where they lie, spatially, in the two-dimensional array. To ensure conservation of flux, it is important that there should be no elevation hollows, where flux can flow in but not out. To avoid this, elevation "polishing" is carried out. This involves identifying and smoothing 
hollows by adding a small elevation to the points within the hollow. This procedure does not affect the pattern of flow but ensures conservation of flux (Budd and Warner, 1996).

The accuracy of the calculated balance velocity is dependent upon the quality of the input data and the method used for calculating them. Equations (1) and (2) indicate that errors in $U_{\mathrm{b}}$ are proportional to errors in accumulation and inversely proportional to errors in ice thickness. Errors in slope aspect have a regional impact as they affect the area, $S$, over which the integration of $A$ takes place, and there is therefore no direct relationship between these errors and the uncertainty in $U_{\mathrm{b}}$. A reasonable approximation, however, is that errors in area scale linearly with errors in $U_{\mathrm{b}}$. Slope aspect errors also have a local effect on the convergence or divergence of flux at any gridpoint, and can therefore influence the local pattern of flow even if the flux, integrated over the entire area, is correct.

Balance velocities reproduce the present-day, depth-averaged velocity if, and only if, (i) there are no errors in the input datasets, (ii) the drainage basin is in balance, (iii) there is time-invariant flow and (iv) the ice flow obeys the dynamical assumptions used in the calculation of $U_{\mathrm{b}}$. Here, we will consider the role of each of these factors in determining the reliability of the balance velocities used in this study.

\section{Input datasets}

Three input datasets are required to estimate $U_{\mathrm{b}}$ : surface slope, ice thickness and mean net surface mass balance. A DEM, containing information on ice surface slope, was derived from ERS-1 satellite radar altimetry and terrestrial data (Bamber and Bindschadler, 1997). For slopes less than about $1^{\circ}$, the bias in the DEM is typically $<10 \mathrm{~m}$ (Bamber and others, 1998). This equates to an error in slope, over $5 \mathrm{~km}$, of about $10 \%$. The slopes for TWG are $<1^{\circ}$ (Fig. 2a). This is also the case for the central part of PIG, although there are higher slope areas (up to $1.5^{\circ}$ ) near the boundaries of the glacier (Fig. 2a). The accuracy of the DEM in the area of interest was examined using surface elevation data obtained from two of the RES flight-lines shown in Figure 1. The positions of the flight-lines have been plotted on a shaded relief plot of surface elevation (Fig. 2b) to indicate their position in relation to the local topography. The data for PIG were collected in 1998 using kinematic global positioning system for navigation control and should be accurate to $\pm 5 \mathrm{~m}$. The data for TWG were flown during the 1970s, and the accuracy of the navigation for these data is considerably poorer. Accuracy in elevation decreases with distance inland, and is typically on the order of several tens of metres. Figure $3 \mathrm{a}$ shows the comparison between the DEM and the airborne elevation measurements on PIG. There is good agreement for the floating tongue, but the break in slope in the DEM precedes the position indicated by the airborne data by about $10 \mathrm{~km}$. This flight-line lies close to the lefthand margin of PIG where the surface slope is at its steepest (Fig. 2b). Figure 3b shows the comparison for TWG, and it is apparent that there is relatively good

\footnotetext{
1 This approximation is inappropriate for floating ice since longitudinal stresses act to smooth out velocity gradients, so the comparison between the InSAR velocities and $U_{\mathrm{b}}$ was restricted to the grounded parts of PIG and TWG.
}

agreement along the length of the profile, which crosses the glacier perpendicular to the flow direction as well as downglacier (Fig. 2b).

Errors in elevation are spatially variable and are difficult to relate directly to errors in $U_{\mathrm{b}}$. However, based on the lower slopes around TWG (Fig. 2a) and the comparison shown in Figure 3, we believe the accuracy of the DEM, in the vicinity of the fast-flowing ice, is better for TWG than for PIG. It is probably comparable for the inland catchment areas, where the slopes are similar and low in both basins. We have assumed that the uncertainty in the area, $A$, over which the integration in Equation (3) takes place is $10 \%$. This is based on a comparison between the area of the PIG drainage basin derived from the DEM used here and a different one (Vaughan and others, 2001).

The presence of longitudinal stresses in the ice can result in local changes to the ice-flow direction. Theory suggests, however, that over distances of 10-20Z, the effect of longitudinal stresses is averaged out, and it can be assumed that ice flows in the direction of the steepest slope (Paterson, 1994). This assumption is made in the calculation of $U_{\mathrm{b}}{ }^{1}$, as discussed earlier. As a consequence, it was necessary to smooth the DEM to reduce the influence of longitudinal stresses on the flow direction. The effect of the smoothing distance on the balance velocities is to redistribute flow on scales similar to the smoothing distance (see, e.g., Bamber and others, 2000a, fig. 8). The DEM was smoothed over a spatially variable distance of $20 Z$. This value was found to provide the best agreement between $U_{\mathrm{b}}$ and InSAR-derived velocities in Greenland (Bamber and others, 2000a). The true scale over which longitudinal stresses can be ignored, however, is a function of the ice rheology and basal traction, which are spatially variable quantities. There is, therefore, no single length scale or factor of ice thickness that will be correct everywhere.

The ice-thickness grid was obtained from a new compilation of data known as BEDMAP (Lythe and others, 2001) and is an improvement on the data used to calculate balance velocities previously published for Antarctica (Budd and Warner, 1996; Bamber and others, 2000b; Huybrechts and others, 2000). Ice-thickness measurements were obtained from airborne RES surveys, supplemented with groundbased spot measurements from seismic soundings (Fig. 1). Both glaciers have RES flight-lines along their length and at least one cross-profile. Ice thickness is also relatively well constrained near the grounding line, based on an inversion of surface elevation, assuming hydrostatic equilibrium (Lythe and others, 2001). The accuracy of $Z$ calculated in this way is about $20 \%$. The RES data have an accuracy within the range $1-10 \%$, dependent primarily on the accuracy of the navigation. This, in turn, is dependent on when the data were collected, with data from the 1970s having the poorest navigational accuracy. For PIG, the RES coverage comprises a mixture of high-accuracy, recently acquired data and older, less accurate data. For TWG, coverage is provided by 1970s data only (Lythe and others, 2001).

It is not only the quality of the raw data, but also its spatial density, that determines the accuracy of the gridded dataset. The accuracy of gridpoints is therefore dependent on their proximity to measurements. Points that are distant from any measurements could have relatively large errors. For the areas of relevance here (in and around the fasterflowing parts of the glaciers) the maximum distance to a measured or estimated ice thickness is about $30-40 \mathrm{~km}$. The mean bedrock slope and ice thickness for the area cov- 

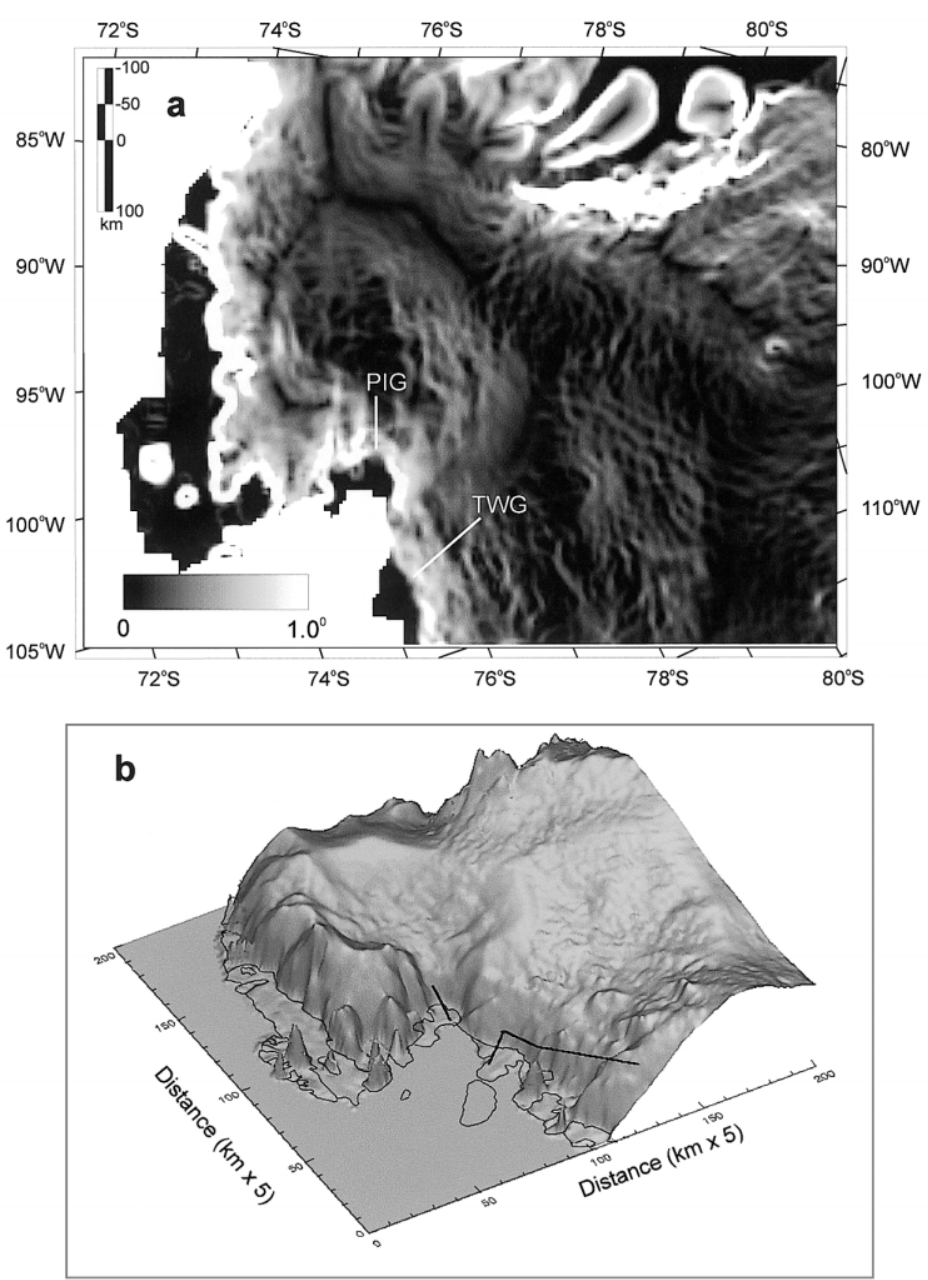

Fig. 2. (a) Surface slopes of 0 $-1^{\circ}$ derived from a $5 \mathrm{~km}$ spacing DEM of the ice sheet (Bamber and Bindschadler, 1997). (b) Shaded relief plot of surface elevation covering PIG and TWG. The locations of the elevation profiles plotted in Figure 3 are indicated by the thick solid lines.

ered by the interferometry are $1.4^{\circ}$ and $1205 \mathrm{~m}$, respectively. A trough or peak with this mean slope and a width of $30 \mathrm{~km}$ would have an amplitude of $367 \mathrm{~m}$, which is $30 \%$ of the mean thickness. The rms of this and the random error in each thickness measurement is $32 \%$. The error in $Z$ varies, therefore, between about $10 \%$ and $32 \%$ depending on the proximity to a measurement. We have used the average of these two limits $(21 \%)$ as an approximation for the mean error in $Z$ over the area covered by the interferometry.

The grid of mean net surface mass balance was obtained from a recent compilation that combined in situ and passivemicrowave satellite measurements (Vaughan and others, 1999). Uncertainty in the surface mass balance over the whole ice sheet is estimated to be 5\% (Vaughan and others, 1999). Much of the ice-core data used to estimate accumulation rates lies in West Antarctica, with a relatively high concentration of points in the Pine Island and Thwaites basins (Vaughan and others 1999, fig. 4). There is, however, a strong gradient in accumulation rate between the coast and inland areas, particularly for the Pine Island basin. For this basin, therefore, we have assumed that the uncertainty in $A$ is $10 \%$. For Thwaites, there are fewer in situ data (Fig. 1) but still substantially more than the average for the continent as a whole. As a consequence, we have assumed that the uncertainty in $A$ for the Thwaites basin is $15 \%$.

Based on the uncertainties in the three input datasets discussed above, the rms error in $U_{\mathrm{b}}$ for PIG and TWG, due solely to errors in the input data, is $25 \%$ and $28 \%$, respectively. It should be emphasized that these are mean values for the areas of interest and that there is considerable spatial variability about these means.

\section{Steady-state flow assumption and time constants}

$U_{\mathrm{b}}$ is a time-averaged quantity. The approximate time constant for the response of a drainage basin in Antarctica is typically on the order of 10000-20000 years (Paterson, 1994). This is approximately the time it would take the topography to relax to a new equilibrium condition given an instantaneous change in boundary conditions or inputs. Locally, the relaxation time is inversely proportional to the velocity. Thus, faster-moving flow features within a basin (such as an ice stream) will have a considerably shorter time constant than the average for an entire basin. Balance velocities are therefore representative of the depth-averaged flow over a period determined by a variable relaxation time on the scale of hundreds to tens of thousands of years. Measured InSAR velocities, however, are much shorter-term surface values and are subject to possible variability due, for example, to changes in basal lubrication. Differences between $U_{\mathrm{b}}$ and a measured velocity can be due, therefore, to either a short-term change in the flow behaviour or a mass imbalance due to a long-term change in the flow and/ or accumulation rates. In Antarctica, no observations of significant short-term (i.e. diurnal to seasonal) variability have been reported. It is unlikely, therefore, that any discrepancy between InSAR and balance velocities will be due to shortterm variability in motion. 

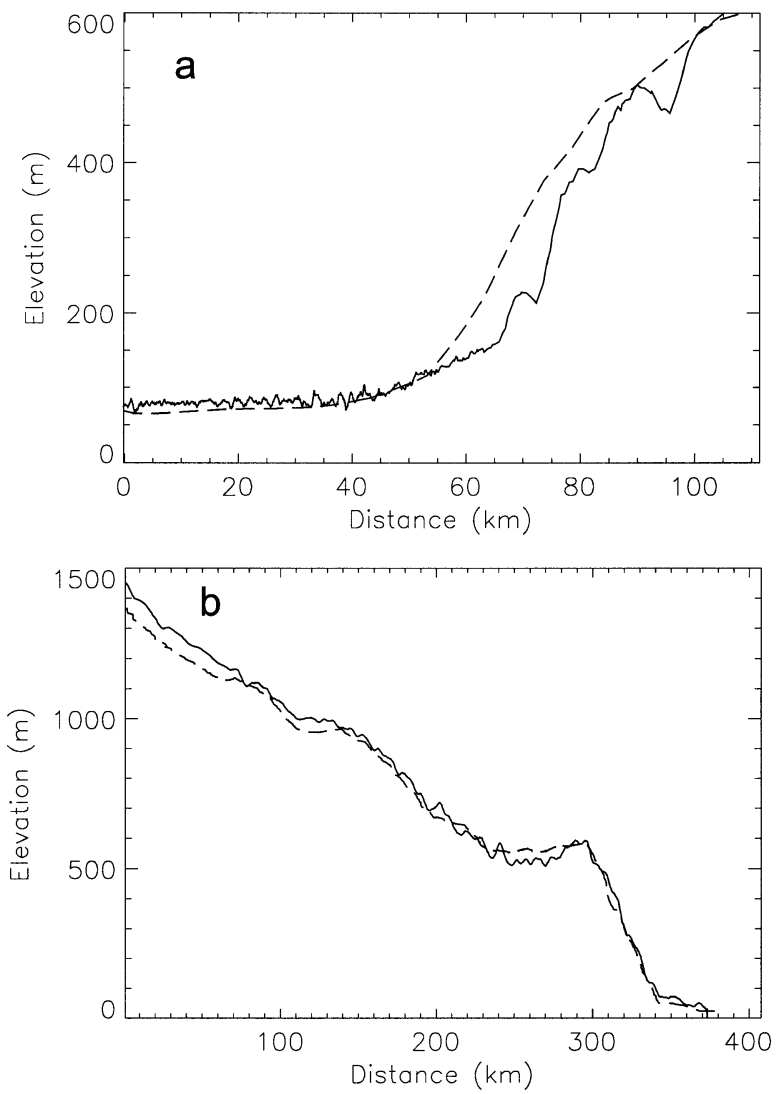

Fig. 3. (a) Comparison of elevations on PIG. The solid line is from airborne RES measurements, and the dashed line shows elevations interpolated from the DEM. (b) As for (a), but for TWG. The locations of both profiles are shown in Figures $2 b, 4$ and 5 .

The ability of balance velocities to reproduce both the pattern and magnitude of flow in Antarctica and Greenland has been demonstrated in two previous studies (Bamber and others, 2000a,b). For the Antarctic study, the comparison between $U_{\mathrm{b}}$ and the InSAR-derived velocities ( $\left.U_{\text {insar }}\right)$ highlighted areas that showed both good agreement, and significant disagreement due to a known change in flow that took place about 130 years ago. This study demonstrated that (i) good agreement could be obtained in areas of presumed steady flow, and (ii) significant (in terms of the rms errors) differences between $U_{\mathrm{b}}$ and $U_{\text {insar }}$ can be used to infer a relatively recent change in flow regime that is reflected in the surface velocity field but not in the topography, which controls the pattern of flow estimated for $U_{\mathrm{b}}$.

\section{InSAR velocities $\left(U_{\text {insar }}\right)$}

ERS SAR data collected in late 1995 to early 1996 during the ERS tandem mission were combined to produce a velocity map of the lower reaches of PIG (Rignot, 1998b) and TWG (Rignot, 2001). Ascending and descending tracks of these data were combined to resolve the vector flow of the glaciers. The process involves correcting the ERS interferograms for the glacier topography, which was estimated by combining differential interferometry data with a DEM of the ice sheet (Bamber and Bindschadler, 1997).

Velocity mapping presents two major difficulties in this region: (1) phase unwrapping and (2) velocity reference. Phase unwrapping (the process of counting phase cycles from a reference zero phase) is difficult because of the rapid rates of surface deformation of the glaciers. It was impossible to unwrap the 19926 day interferograms on PIG or TWG. Reference points of known velocity are required to provide an absolute reference for the InSAR measurements and to ensure that there are no residual gradients in the velocity map. In the case of PIG, the area of fast flow is relatively well confined, surrounded by numerous volcanoes and mountain peaks emerging from the surrounding ice (Fig. 1). This makes zero-referencing easy, provided that a large enough area is processed along each ERS track, i.e. several $100 \mathrm{~km}$ long frames. In the case of TWG, control was provided by a mountain range west of the glacier, and an isolated ice dome near the coast to the east (Fig. 1).

InSAR surface velocities for TWG were initially produced using the DEM of Antarctica, and subsequently revised using an InSAR-derived DEM of the region (from the same data). We found a difference in ice velocity of $5 \%$ between the two maps because of the better control with the InSAR DEM on areas of no motion (the Antarctic DEM was relatively poor over our areas of control, as these are isolated outcrops with large, short-wavelength relief). In areas where we have control points, we estimate that the accuracy of the velocities for the two glaciers made from ERS data acquired in 1996 is typically about $10 \mathrm{ma}^{-1}$. Inland, away from the areas of control, the errors may be higher than this. In some other areas, phase unwrapping could not be performed, resulting in gaps in the derived velocity field (shaded black in Figs $4 \mathrm{a}$ and $5 \mathrm{a}$ ).

\section{RESULTS}

$U_{\text {insar }}$ and $U_{\mathrm{b}}$ are plotted in Figure $4 \mathrm{a}$ and $\mathrm{b}$ for PIG, and in Figure $5 \mathrm{a}$ and $\mathrm{b}$ for TWG. The grounding lines were determined from the InSAR data using a multiple differencing approach (Rignot, 1998a). Balance velocities beyond the grounding line are not plotted, for reasons discussed earlier. Figure 4 indicates that the pattern of motion is in broad agreement for PIG, although the area of rapid flow in the upstream portion in Figure $4 \mathrm{~b}$ is too narrow. This is most probably due to the relatively steep relief in this upstream area, particularly at the margins of the glacier (Fig. 2b), which has resulted in a narrower trough in the DEM than actually exists. This is a known limitation of satellite radar altimetry over undulating terrain: the peaks are sampled more frequently than the troughs, resulting in a positive bias within the troughs (Bamber, 1994; Bamber and others, 2001). This effect is illustrated in Figure 3a. Despite this limitation, the general pattern of flow is captured well, including the tributaries (marked $\mathrm{T} 1$ and $\mathrm{T} 2$ ) flowing into the upstream end of PIG and near the northern margin of the grounding line. The relatively low slopes and good agreement shown in Figure $3 \mathrm{~b}$ indicate that the errors in topography for TWG are considerably smaller than for PIG, yet about half the entire area covered by interferometry over TWG is flowing substantially slower for the $U_{\mathrm{b}}$ estimate compared with the measured velocities $\left(U_{\text {insar }}\right)$. This is illustrated in Figure 6, which shows the difference between $U_{\mathrm{b}}$ and $U_{\text {insar }}(\Delta U)$ over the grounded part of the glacier. It is clear that for a large swathe of the central part of the glacier, roughly

2 Ice thickness is not required in calculating a balance flux, so the error in this term is less than for a balance velocity. 

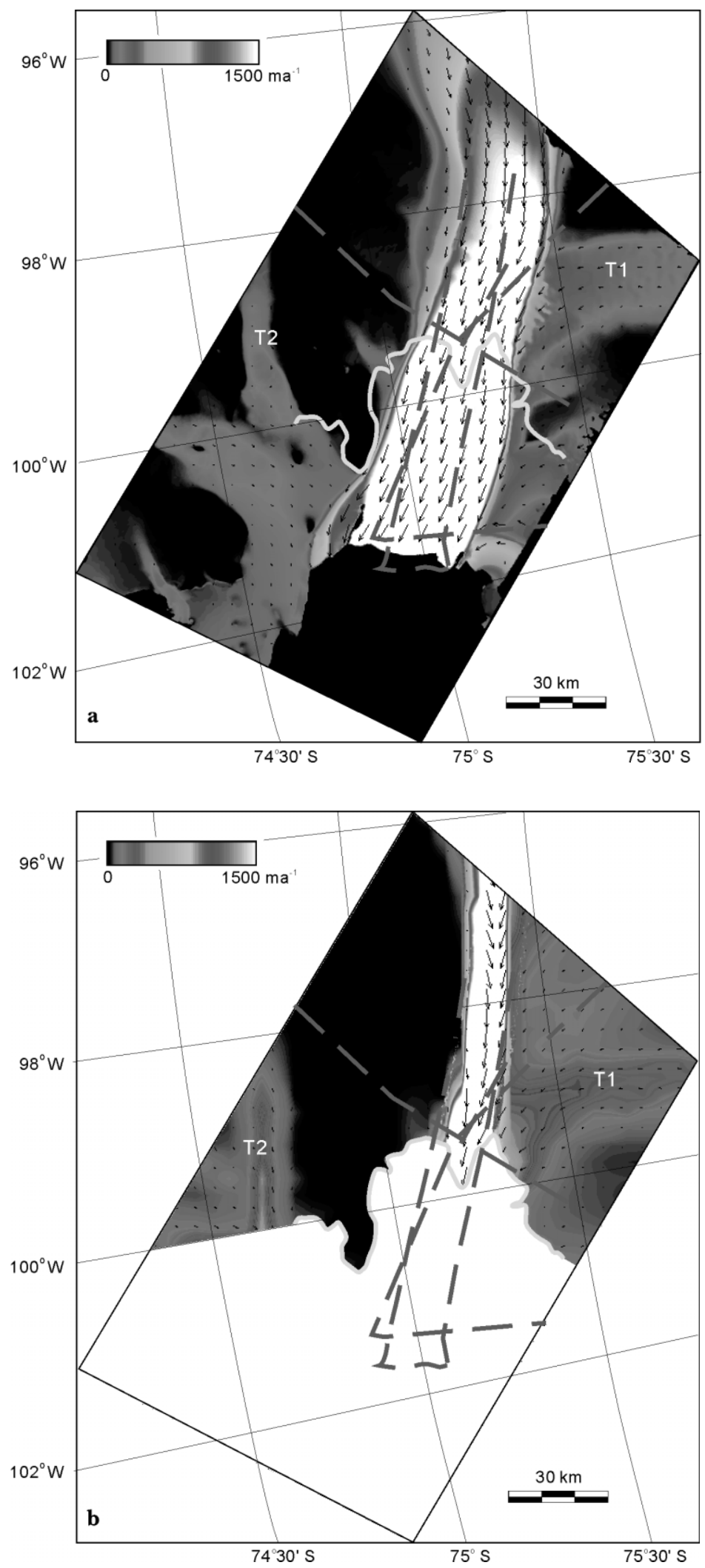

Fig. 4. Plots of ( a) InSAR velocity $\left(U_{\mathrm{insar}}\right)$ and $(b)$ balance velocity $\left(U_{\mathrm{b}}\right)$ for PIG. The direction and magnitude of flow is indicated by the black arrows, with the maximum length equating to a velocity of $1000 \mathrm{~m} \mathrm{a}{ }^{-1}$. The grounding line, as determined by interferometry and used to estimatefluxes, is shown as a solid blue line. The locations of ice-thickness flight-lines are shown by the green lines. The dashed sections indicate the locations of the elevation profiles plotted in Figure 3. Ice thickness was also determined close to the grounding line, based on the assumption of hydrostatic equilibrium and a knowledge of the surface elevation and densities of sea water and ice. The black areas within the area of coverage of the SAR data are where it was not possible to obtain an interferometrically derived velocity.

between $105^{\circ}$ and $108^{\circ} \mathrm{W}, U_{\text {insar }}$ is substantially greater than $U_{\mathrm{b}}$ (by $>1000 \mathrm{~m} \mathrm{a}^{-1}$ for the central "core"). The width and length of this region is about $70 \mathrm{~km}$, covering all of the fastest-flowing part of the glacier shown in Figure $5 \mathrm{a}$ and an extensive region east of this.
Also of importance is the fact that the peak in flow occurs further west (lower) for $U_{\mathrm{b}}$. This is illustrated graphically in Figure 7, which is a plot of velocity profiles for $U_{\mathrm{b}}$ and $U_{\text {insar }}$ along the grounding lines of the two glaciers. Again, there is broad agreement in the pattern of flow for 

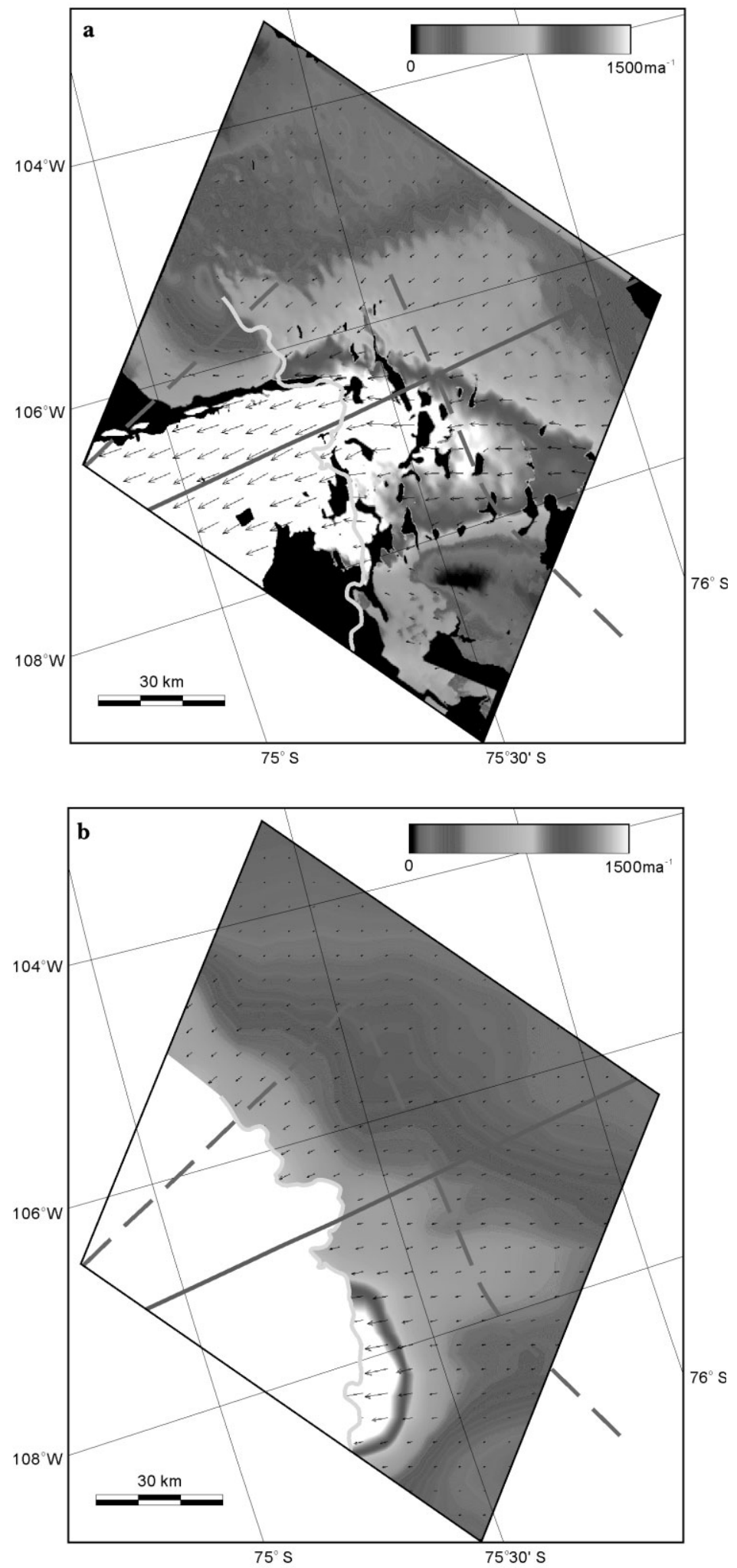

Fig. 5. ( $a, b)$ As for Figure 4, but for TWG. The maximum length of the arrowe is equivalent to a velocity of $2000 \mathrm{~m} a^{-1}$.

the central part of PIG, with $U_{\mathrm{b}}$ being too narrow, as mentioned earlier, resulting in a higher peak velocity. For TWG, although there is better agreement in the magnitudes, the position of peak flow is displaced by about $50 \mathrm{~km}$. Interestingly, the two maxima in flow observed for $U_{\text {insar }}$ are also seen for $U_{\mathrm{b}}$. Balance fluxes were calculated across the grounding lines, and agreed with the most recent previous estimates within 5\%, although they were estimated using a different methodology. The balance flux for PIG was $9 \pm 14 \%{ }^{2}$ less than the measured outgoing flux, while for TWG it was $35 \pm 22 \%$ less.

\section{INTERPRETATION AND DISGUSSION}

\section{Errors in balance velocities}

The ice-thickness coverage includes profiles running downflow on each glacier, as well as a single profile across TWG and several across PIG (Figs 1, 4 and 5). The thickness near the grounding lines was constrained assuming hydrostatic equilibrium for the floating ice and converting surface elevation to ice thickness. Nonetheless, significant interpolation was required to produce the regularly spaced $5 \mathrm{~km}$ grid pro- 


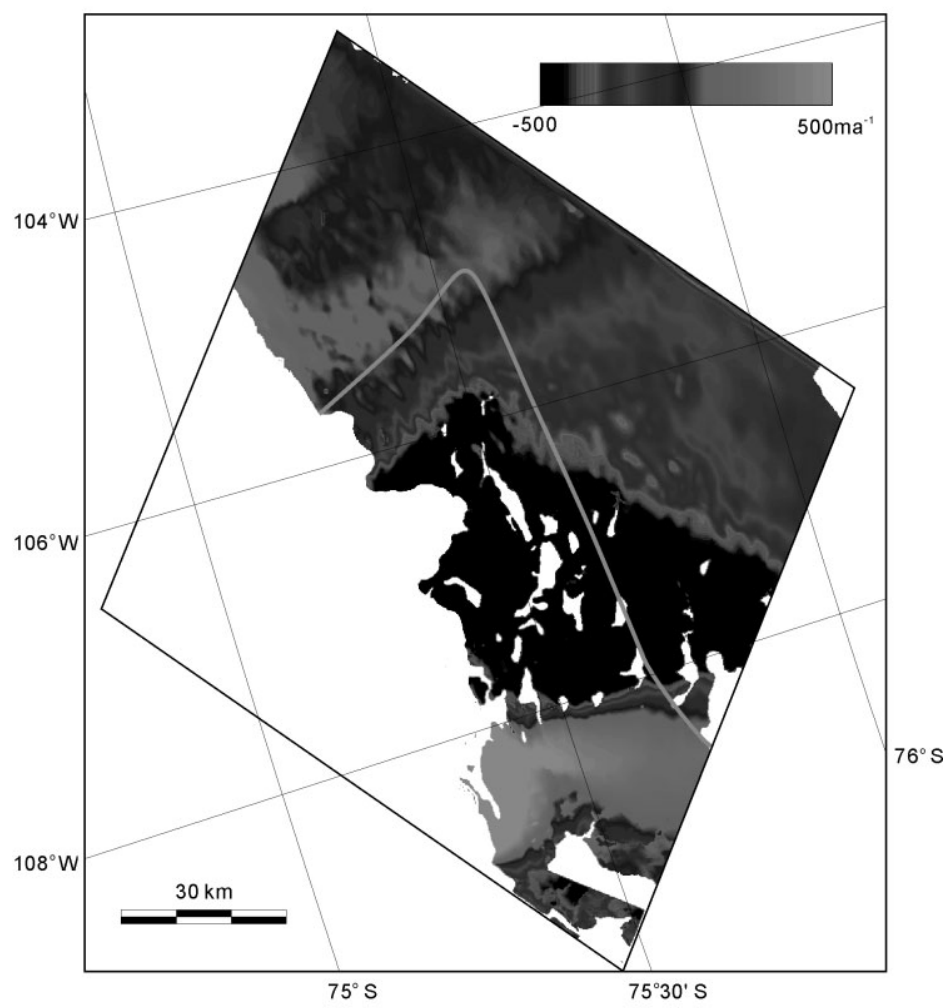

Fig. 6. The difference in velocity, $\Delta U\left(U_{\mathrm{b}}-U_{\text {insar }}\right)$, for the grounded part of $T W G$, ranging from -500 to $500 \mathrm{ma} a^{-1}$. The RES flight-line, along which $U_{\mathrm{b}}$ and $U_{\mathrm{insar}}$ are compared in Figure 8, is shown by the solid yellow line. Areas shaded white are where there were no valid velocity data for $U_{\mathrm{b}}$ and/or $U_{\text {insar. }}$

duced by the BEDMAP consortium, especially for TWG (Lythe and others, 2001). Accumulation data are more sparse, especially for the basin containing TWG. Errors in these two parameters scale linearly with $U_{\mathrm{b}}$. Thus, a $10 \%$ underestimate in thickness results in a $10 \%$ increase in the estimated value of $U_{\mathrm{b}}$. It is clear that errors in $Z$ cannot explain the velocity differences seen at the grounding line of TWG (Fig. 7c). To investigate whether this conclusion was also supported inland, $U_{\mathrm{b}}$ and $U_{\text {insar }}$ were compared along part of a RES flight-line (shown in Fig. 6), where $Z$ is well constrained. The results of this comparison are shown in Figure 8. The very large difference (of as much as a factor 3 ) between $U_{\mathrm{b}}$ and $U_{\text {insar }}$ from $10-50 \mathrm{~km}$ along track clearly cannot be explained by errors in $Z$. Interestingly, there is good agreement between $U_{\mathrm{b}}$ and $U_{\text {insar }}$ from $100-150 \mathrm{~km}$ along-track, in the area outside of the fast-flowing ice. This coincides with the area of green and orange between about $103^{\circ}$ and $105^{\circ} \mathrm{W}$ in Figure 6. This good agreement supports the conclusion that it is not errors in the input datasets that cause the large differences between $U_{\mathrm{b}}$ and $U_{\text {insar }}$ but that a fundamental change in the flow regime is probably responsible. Given the coverage of ice-thickness data, and noting that $Z$ is also well constrained near the grounding line (where large values of $\Delta U$ are found), it is difficult to see how errors in $Z$ can explain the large differences seen in Figure 6.

It is unlikely that errors in accumulation rate are responsible for the large differences in velocity, as this is a relatively slowly varying parameter spatially, and what is required is a large $(>300 \%)$ and variable error over distances of tens of kilometres. Short-wavelength errors in surface slope could produce an offset in the position of peak flow, but TWG is in an area of relatively low surface slope (Fig. 2a) and low errors (Fig. 3b) with good coverage by satellite radar altimeter data, so this is also an unlikely explanation. It is thus difficult to explain the poor agree- ment in both the pattern and magnitude of flow for TWG by errors in the input datasets.

The area of negative differences for TWG cannot be explained by the variable influence of longitudinal stresses (i.e. a breakdown in the assumptions about flow). The area is spatially extensive and smoothly increasing from a minimum near the grounding line. One final possibility is that the ratio of depth-averaged to surface velocity varies dramatically but this is not supported by evidence from thermomechanical models of ice-sheet flow (Bamber and others, 2000a), which predict a ratio of $0.87-1.0$ for the Greenland ice sheet. The value of this ratio is generally assumed to be close to 1.0 at the grounding line, yet, as mentioned, large values of $\Delta U$ are found here.

\section{Errors in InSAR velocities}

Velocities from descending tracks of SAR data for TWG are well constrained because they include areas of no ice motion (volcanoes, coastal peaks). The ascending tracks covering TWG have a longer interferometric baseline, which is poorer for velocity mapping, and do not have such reliable velocity control east of PIG. Control was only provided over the $50 \mathrm{~km}$ long sector in the lower part of the track (Fig. 1). Overall, however, and based on improvements of the velocity map compared to an earlier map, we estimate that ice velocity is measured with an accuracy of $10-20 \mathrm{~m} \mathrm{a}^{-1}$ over PIG and most of TWG, as discussed earlier.

\section{State of balance}

A short-term, historical change in accumulation rate (rather than an error in it) would cause the estimated balance flux (and consequently $U_{\mathrm{b}}$ ) to be different from the present-day surface velocities. As discussed above, a very substantial, complex and implausible pattern of decrease in 

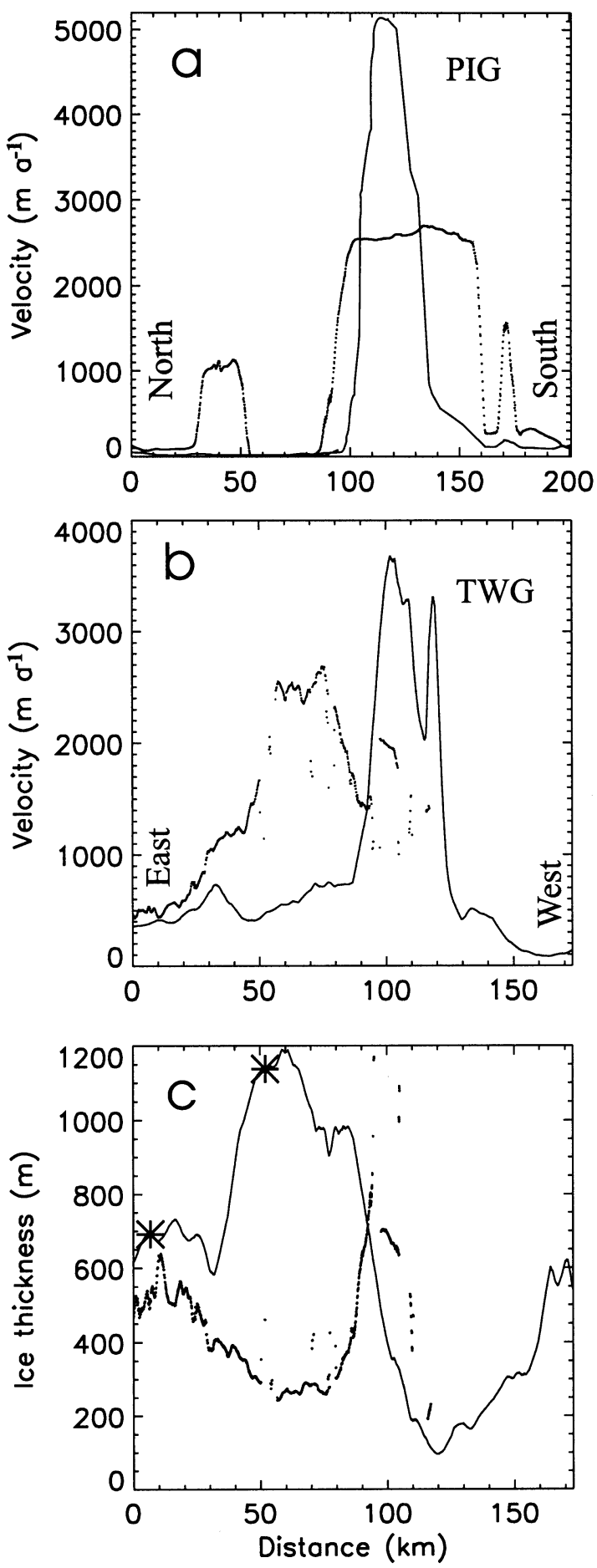

Fig. 7. InSAR (points) and balance velocities (solid line) for the grounding lines of (a) PIG and (b) TWG. The lines run north-south and east-west, respectively. (c) Ice thickness along the grounding line derived from the BEDMAP dataset (solid line). The two crosses indicate the position of measured thicknesses. The dots indicate the thickness that would be required to make the balance velocities match the InSAR values.

accumulation over much of the area covered is required, however. There is no evidence of such a change in accumulation rates from either ice cores or general circulation model simulations of the climate in Antarctica over the last century. Furthermore, the recently determined imbalance in PIG has been associated with a change in flow regime and not accumulation (Shepherd and others, 2001). We believe, therefore, that a localized change in accumulation rate cannot fully explain the large differences seen in Figures 5, 6 and 8.

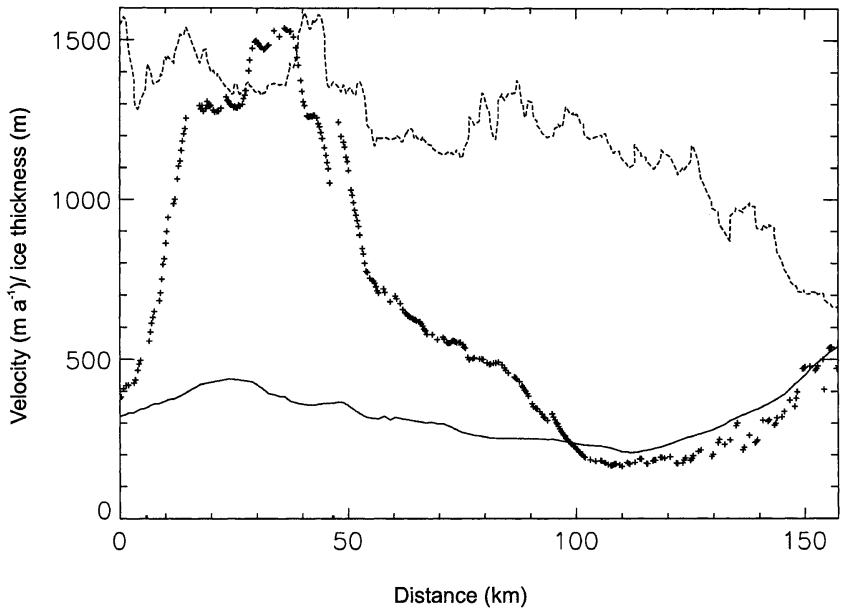

Fig. 8. A graph of balance velocity (solid line) and InSARderived velocity (crosses) along the flight-line shown in Figure 6 for TWG. Also plotted is the measured ice thickness (dashed line). The track is plotted south-north, and the change in direction at about $75.5^{\circ} \mathrm{S}, 105^{\circ} \mathrm{W}$ takes place about $110 \mathrm{~km}$ along-track.

\section{Time-invariant flow}

The absolute difference in velocities for TWG (Fig. 6) indicates that the largest differences $\left(>1000 \mathrm{ma}^{-1}\right)$ could be reduced if the peak flow in $U_{\mathrm{b}}$ were shifted further east (upwards in Fig. 6). The discrepancy, however, covers an area of about $5000 \mathrm{~km}^{2}$, and so cannot be due to the smoothing distance used in the balance-velocity calculation or to shortwavelength errors in the ice-thickness data due, for example, to an undetected, subglacial feature. The cause of the discrepancy is "broad-scale", affecting a large part of the grounding line and inland areas where ice thickness is well constrained (Figs 7 and 8).

We believe the most plausible explanation for the differences observed for TWG is a spatially extensive alteration in the flow of the glacier and surrounding ice. We propose that the glacier may have accelerated in the last few centuries, producing a mismatch between present-day observations of velocity and ones which represent flow over a longer timescale. This inference is similar to that obtained when $U_{\mathrm{b}}$ and $U_{\text {insar }}$ were compared for Ice Stream C (Bamber and others, $2000 \mathrm{~b})$. In this case, however, it was already known that the ice stream had recently shut down $(\sim 130$ years BP $)$ and what was observed was the converse of the difference seen on TWG: the surface velocities from InSAR were those associated with creep flow (on the order of $50 \mathrm{~m} \mathrm{a}^{-1}$ ), whereas the balance velocities produced values of $\sim 250 \mathrm{ma}^{-1}$ (i.e. $U_{\mathrm{b}}$ was a factor five greater than $U_{\text {insar }}$ ).

\section{GONCLUSIONS}

Balance velocities were compared with InSAR-derived values for Pine Island and Thwaites Glaciers. No statistically significant difference between the two measures of velocity was detected for PIG. An estimation of the grounding-line discharge of the glacier suggested that PIG was losing mass by $9 \pm 14 \%$ in 1996 . As the glacier accelerated $10 \%$ between 1996 and 2000, the same comparison repeated in 2000 would probably show a mass loss above the level of uncertainty. Nonetheless both the pattern of flow and total flux of ice crossing the grounding line were in broad agreement.

A different situation was found for TWG. The most likely explanation for the pattern and magnitude of the observed 
differences is a substantial change in flow behaviour during the recent past. We note, however, that no change in velocity was observed between the 1970s and 1990s (Rosanova and others, 1999) using feature-tracking techniques, nor from 1996 to 2000 (Rignot and others, 2002) using InSAR. This suggests that the velocity of the glacier has not changed dramatically in the last few decades. Yet the InSAR data of Rignot and others (2002) imply that the ice stream may be widening with time, with the result that ice discharge increased by $4 \%$ in 4 years. Hence, while the bulk velocity of TWG has not changed at a detectable level $(1 \%$ in 4 years with InSAR), the glacier may not yet have reached a steady state. Thus it is possible that TWG is still in a transition stage. This is consistent with the fact that it is significantly out of balance with the interior accumulation at present. Here, we found that the grounding-line flux, as measured using InSAR, was $32 \pm 19 \%$ greater than that required for balance. This finding is supported by the $10 \mathrm{~cm} \mathrm{a}^{-1}$ decrease in surface elevation obtained from ERS radar altimetry over the drainage basins of PIG and TWG (Wingham and others, 1998). This is equivalent to an imbalance of $24 \%$ based on the mean accumulation rate for the two basins (Vaughan and others, 1999). While the thinning rate was interpreted, at that time, as a change in accumulation rate, the results of this study and other more recent work on PIG and TWG suggest instead that the change in ice volume is related to changes in flow regime and has, as a consequence, potentially profound implications for the future of this part of the West Antarctic ice sheet.

\section{AGKNOWLEDGEMENTS}

The authors would like to thank the European Space Agency for provision of the SAR data, R. C. Warner of Antarctic CRC for provision of the balance-velocity code, D. G. Vaughan of the British Antarctic Survey for drafting Figure 1, and R. A. Bindschadler, I. R. Joughin and T. A. Scambos for their constructive comments and editorial efforts with the various versions of this paper. This work was partfunded by U.K. Natural Environment Research Council grants GR3/EOCE/4 and GR3/AFI/65.

\section{REFERENCES}

Bamber, J. L. 1994. Ice sheet altimeter processing scheme. Int. F. Remote Sensing, 15(4), 925-938.

Bamber, J. L. and R. A. Bindschadler. 1997. An improved elevation dataset for climate and ice-sheet modelling: validation with satellite imagery. Ann. Glaciol., 25, 439-444.
Bamber, J. L., S. Ekholm and W. B. Krabill. 1998. The accuracy of satellite radar altimeter data over the Greenland ice sheet determined from airborne laser data. Geophys. Res. Lett., 25(16), 3177-3180.

Bamber, J. L., R. J. Hardy and I. Joughin. 2000a. An analysis of balance velocities over the Greenland ice sheet and comparison with synthetic aperture radar interferometry. F. Glaciol., 46(152), 67-74.

Bamber, J. L., D. G. Vaughan and I. Joughin. 2000b. Widespread complex flow in the interior of the Antarctic ice sheet. Science, 287(5456), 1248-1250.

Bamber, J. L., S. Ekholm and W. B. Krabill. 2001. A new, high-resolution digital elevation model of Greenland fully validated with airborne laser altimeter data. 7. Geophys. Res., 106(B4), 6733-6746.

Bentley, C. R. and M. B. Giovinetto. 1991. Mass balance of Antarctica and sea level change. In Weller, G., C. L. Wilson and B. A. B. Severin, eds. International Conference on the Role of the Polar Regions in Global Change: proceedings of a conference held June 11-15, 1990 at the University of Alask a Fairbanks. Vol. II. Fairbanks, AK, University of Alaska. Geophysical Institute/Center for Global Change and Arctic System Research, 481-488.

Budd, W. F. and R. C. Warner. 1996. A computer scheme for rapid calculations of balance-flux distributions. Ann. Glaciol., 23, 21-27.

Crabtree, R. D. and G. S. M. Doake. 1982. Pine Island Glacier and its drainage basin: results from radio-echo sounding. Ann. Glaciol., 3, 65-70.

Hughes, T. J. 1981. Correspondence. The weak underbelly of the West Antarctic ice sheet. F. Glaciol., 27(97), 518-525.

Huybrechts, P., D. Steinhage, F. Wilhelms and J. Bamber. 2000. Balance velocities and measured properties of the Antarctic ice sheet from a new compilation of gridded data for modelling. Ann. Glaciol., 30, 52-60.

Lucchitta, B. K., C. E. Smith, J. A. Bowell and K. F. Mullins. 1994. Velocities and mass balance of Pine Island Glacier, West Antarctica, derived from ERS-1 SAR images. In Space at the Service of our Environment. Proceedings of the Second ERS-1 Symposium, 11-14 October 1993, Hamburg, Germany. Vol. 1. Paris, European Space Agency, 147-151. (ESA SP-361.)

Lythe, M. B., D. G. Vaughan and BEDMAP Consortium. 2001. BEDMAP: a new ice thickness and subglacial topographic model of Antarctica. 7 . Geophys. Res., 106 (B6), 11,335-11,351.

Paterson, W. S. B. 1994. The physics of glaciers. Third edition. Oxford, etc., Elsevier.

Rignot, E. J. 1998a. Fast recession of a West Antarctic glacier. Science, 281 (5376), 549-551.

Rignot, E. 1998b. Hinge-line migration of Petermann Gletscher, north Greenland, detected using satellite-radar interferometry. 7. Glaciol., 44(148), 469-476

Rignot, E. 2001. Evidence for rapid retreat and mass loss of Thwaites Glacier, West Antarctica. 7. Glaciol., 47(157), 213-222.

Rignot, E., D. G. Vaughan, M. Schmeltz, T. Dupont and D. MacAyeal. 2002. Acceleration of Pine Island and Thwaites Glaciers, West Antarctica. Ann. Glaciol., 34, 189-194.

Rosanova, C. E., B. K. Lucchitta and J. G. Ferrigno. 1999. Thwaites Glacier velocities and acceleration: comparing Landsat and ERS-1 SAR data. [Abstract.] Eos, 80(46), Fall Meeting Supplement, F330.

Shepherd, A., D. J. Wingham, J. A. D. Mansley and H. F. J. Corr. 2001. Inland thinning of Pine Island Glacier, West Antarctica. Science, 291(5505), 862-864.

Vaughan, D. G., J. L. Bamber, M. B. Giovinetto, J. Russell and A. P. R. Cooper. 1999. Reassessment of net surface mass balance in Antarctica. 7. Climate, 12(4), 933-946.

Vaughan, D. G. and 9 others. 2001. A review of Pine Island Glacier basin, West Antarctica: hypotheses of instability vs. observations of change. In Alley, R. B. and R. A. Bindschadler, eds. The West Antarctic ice sheet: behavior and environment. Washington, DC, American Geophysical Union, 237-256. (Antarctic Research Series 77.)

Wingham, D. J., A. L. Ridout, R. Scharroo, R. J. Arthern and C. K. Shum. 1998. Antarctic elevation change 1992 to 1996. Science, 282(5388), 456-458. 